\title{
A Novel 3-Input AND/XOR Gate Circuit for Reed-Muller Logic Applications
}

\author{
Yuan Yang, Yinshui Xia and Libo Qian \\ Faculty of Electrics Engineering and Computer Science, Ningbo University, 315211 Ningbo, Zhejiang, China
}

\begin{abstract}
AND/XOR is the basic complex gate of Reed-Muller logic. Low energy consumption is important for Reed-Muller logic circuit implementation. Against the drawbacks of the published gate-level and transistor-level 3-input AND/XOR gate design in power and power delay product (PDP), a low energy consumption 3-input AND/XOR gate is proposed by employing multi-rails and hybrid-CMOS techniques to improve its speed and short the signal transimission path. Under 55nm CMOS process, post-simulations in different process corners are carried out by using HSPICE and compared with the published circuits. Simulation results show that the proposed circuit has advantages over published designs. For typical process corners, the improvement of the proposed circuit can be up to $27.21 \%, 19.23 \%$ and $35.39 \%$, respectively, in terms of power, delay and power delay product.
\end{abstract}

\section{Introduction}

As the rapid development of design technology and CMOS process shrinking, the design concerns arise to comprehensive requirements of performance, area and power. Currently, almost all of the digital circuits are implemented by the traditional Boolean (TB) logic based on AND, OR and NOT. Actually, digital circuits can also be implemented by Reed-Muller (RM) logic based on AND/XOR. Studies show that statistically half of the circuits implemented by the RM logic can achieve better performance than those by TB logic. Moreover, compared to the TB logic, RM logic has the following advantages: firstly, RM logic circuit is easy to be mapped to the field programmable gate array (FPGA) and has good testability properties [1]-[2], which provide an effective way to solve the problem of "verification" in integrated circuit design; secondly, RM logic is much simpler in the implementation of some logic functions such as arithmetic components and parity function [3], which not only brings about smaller area, but also has the potential advantages of power and speed. However, RM logic was not widely used because of the absence of corresponding complex gate circuits and EDA tools. In recent years, some 3-input AND/XOR gates based on gate level and transistor level are published, but far from satisfaction in terms of power consumption, delay and Power Delay Product (PDP).

In this paper, a novel transistor level based 3-input $\mathrm{AND} / \mathrm{XOR}$ gate design is proposed. Under $55 \mathrm{~nm}$ CMOS process, the post-simulations of the circuit under different process corners are carried out by HSPICE and compared with the published circuits. The simulation results show that the proposed circuit has advantages in power consumption and delay.

\section{Published 3-Input AND/XOR gates}

3 -input $\mathrm{AND} / \mathrm{XOR}$ is the basic complex gate of RM logic that performs the following equation:

$$
A B \oplus C=\overline{A B C}+A B \bar{C}=\overline{A B} \oplus \bar{C}
$$

XOR and OR, denoted by $\oplus$ and + respectively, are binary operations. According to the structure of 3-input AND/XOR, its design method can be divided into two paths: gate level and transistor level.

Gate level-based 3-input AND/XOR gate is implemented by cascading one AND (or NAND) gate and one XOR (or XNOR) gate. In recent years, researchers already place a high value on XOR-XNOR circuits because they are basic building blocks in various circuits especially in Arithmetic circuits, Parity Checks, Error-detecting.

Depending on the difference in XOR gates, various kinds of AND/XOR gate circuits are proposed in Fig. 1 (a)-(c). In Fig. 1 (a), a 3-input $\mathrm{AND} / \mathrm{XOR}$ gate is implemented by cascading one NAND gate and one XOR gate in complementary CMOS structure [4-5], which consists of a pull-up PMOS network and a pull-down NMOS network. As a result, the gate circuit has a symmetrical structure and operates with full output voltage swing, but it requires significant transistors and high power consumption. In Fig. 1 (b), a 3-input AND/XOR gate [6] is implemented by cascading one AND gate and one XOR gate [7] in pass-transistor logic (PTL) structure. PTL allows the source terminal of the MOS transistor is connected to input line. Consequently, the number of transistors and the related parasitic capacitance are reduced. However, PMOS and NMOS have poor performance on transmitting the signal "LOW" 
and "HIGH", respectively, leading to threshold voltage

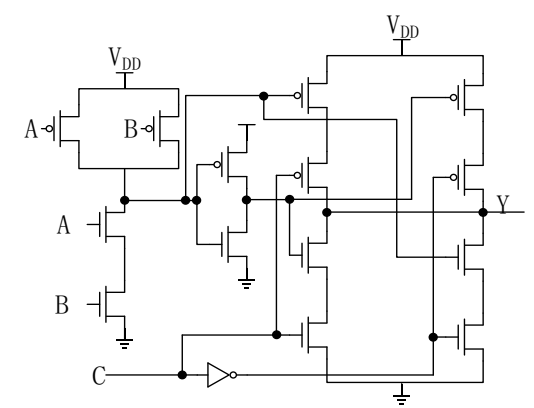

(a)CMOS

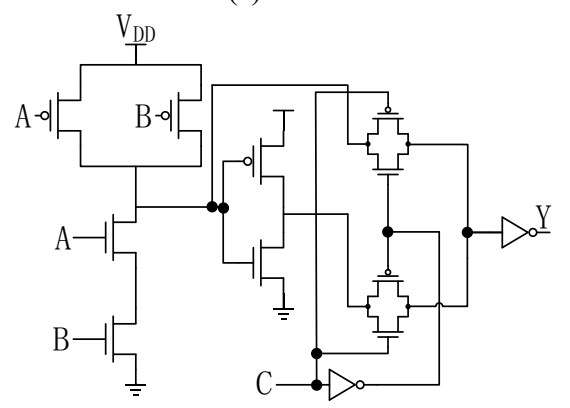

(c) $\mathrm{TG}$

Figure 1. Published 3-Input AND/XOR gates

PTL circuits. To make the XOR gate operate with full voltage swing at all nodes, a transmission gate (TG) is added in the XOR gate [8]. In addition, a static CMOS inverter is cascaded to improve the driving capability of PTL circuits. Based on TG logic, a 3-input AND/XOR gate is implemented by cascading one NAND gate and one XOR gate [9], as shown in Fig. 1 (c). The XOR gate is composed of two transmission gates and three static CMOS inverters. Thus, it has a full voltage swing at all nodes for all input patterns, so that it can be operated at lower supply voltage. Similarly, the inverters enhance the driving capability.

Based on transistor level, a 3-input AND/XOR gate [10] is designed as shown in Fig. 1 (d). It is composed of one XOR gate, two transmission gates and two static CMOS inverters. The input signal $A$ and its complementary input signal control two transmission gates so that only one TG can work at the same time. This design has a full voltage swing at all nodes for all input patterns and negligible short-circuit power dissipation, which lead to lower power consumption. Furthermore, two static CMOS inverters are cascaded after the circuit to improve the driving capability.

Based on the discussion above, the published AND/XOR gates have different disadvantages, the complementary CMOS logic based 3-input AND/XOR gate circuit requires most numbers of MOS transistors and has a high power consumption; the PTL or TG based 3-input AND/XOR gate circuits have long critical paths and the more numbers of internal nodes consume extra power consumption; the transistor level based 3-input AND/XOR gate circuits has a long critical paths. Therefore, the performance of $\mathrm{AND} / \mathrm{XOR}$ has yet to be further improved.

\section{Proposed 3-Input AND/XOR gate}

loss at some nodes with certain input Combinations in

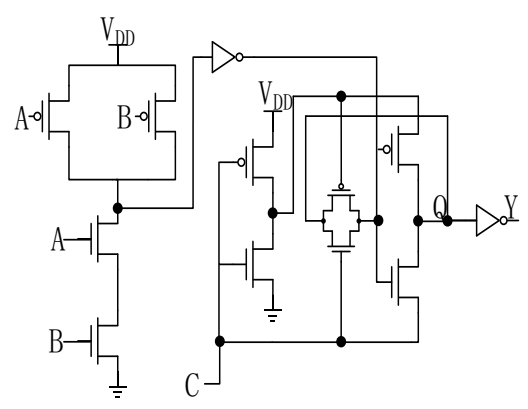

(b) HU12

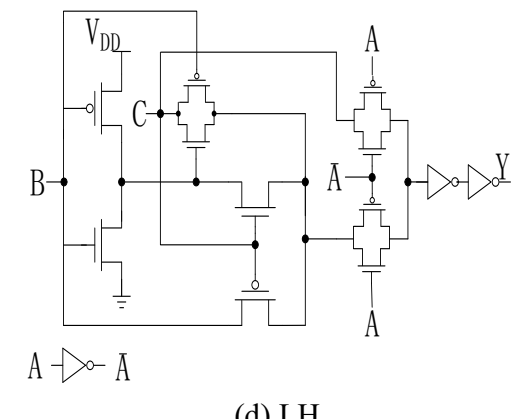

(d) $\mathrm{LH}$

Based on hybrid-CMOS techniques, a 16T 3-input AND/XOR gate circuit is proposed, as shown in Fig. 2 (a). The circuit contains two substructures and has a full voltage swing at all nodes for all input combinations.

The basic function of the gate circuit is as follows. When $\mathrm{AC}=00, \mathrm{P} 1$ and $\mathrm{P} 3$ turn on and pass a strong "HIGH" signal level to node $\mathrm{Q}$ and then a strong "LOW" to the output. When $\mathrm{BC}=00, \mathrm{P} 2$ and $\mathrm{P} 3$ turn on and pass a strong "HIGH" signal level to node $\mathrm{Q}$ and then a strong "LOW" to the output. When $A C=01, \mathrm{~N} 1$ and $\mathrm{N} 2$ turn on and pass a strong "LOW" to node $\mathrm{Q}$ and then a strong "HIGH" to the output. Under five input combinations above, only substructure 1 works. Due to the node $\mathrm{Q}$ is connected to power supply or ground, is has full voltage swing under lower supply voltage, and there is no direct path between power supply and ground, which reduce the power consumption.

When $\mathrm{ABC}=111, \mathrm{~N} 3, \mathrm{~N} 4$ and N5 turn on and pass two weak "HIGH" to the node Q. In order to eliminate threshold voltage loss, the third transmission path, which include P4 and P5, is added, meanwhile, corresponding performance is also improved. When $\mathrm{ABC}=101, \mathrm{P} 4, \mathrm{P} 5$, N3 and N5 turn on and N3 and N5 pass a strong "LOW" to the node $\mathrm{Q}$ and a strong "HIGH" to the output. When $\mathrm{ABC}=110, \mathrm{~N} 3$ and $\mathrm{N} 4$ turn on and pass a strong "LOW" to the node $\mathrm{Q}$ and a strong "HIGH" to the output. Under above three input combinations, only substructure 2 works, and all internal nodes have full voltage swing. Due to multi-rails of structure 2 when $A B C=111$ or 101 , the equivalent resistance of transmission path is smaller, which improve the charge and discharge speed of output, then enhance the working efficiency of the circuit. The cascade inverter is used to strengthen the circuit driving capability. The proposed design is modular and simple, which simplify the layout and optimize chip area potentially. The layout of the proposed design is shown in Fig. 2 (b). 


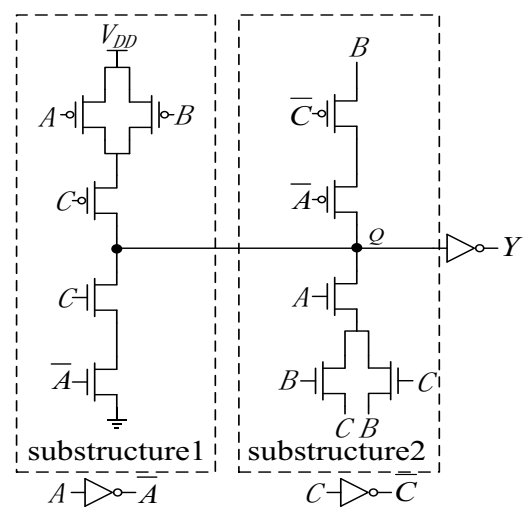

(a) The structure of the proposed AND/XOR gate

Figure 2. Proposed circuit

\section{Simulation and comparison}

The circuit simulation is carried out using HSPICE under $55 \mathrm{~nm}$ CMOS process at $1.20 \mathrm{~V}$ supply voltage. The operating frequency is $100 \mathrm{MHZ}$. The gate area ratio of PMOS and NMOS are about 2:1 while the physical W/L sizes of PMOS and NMOS are $240 \mathrm{~nm} / 60 \mathrm{~nm}$ and $120 \mathrm{~nm} / 60 \mathrm{~nm}$, respectively. To model the realistic environment, the input terminals are cascaded with twostage inverters and the output drives are four parallel inverters. The simulation test bench used is shown in Fig. 3. All possible input combinations at the gate inputs are simulated. The transient waveforms are shown in Fig. 4. It can be seen that the proposed circuit has correct function for all of the input cases.

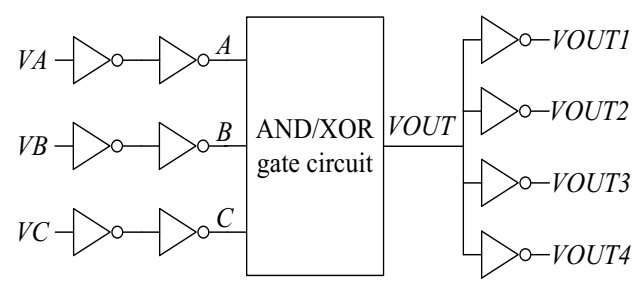

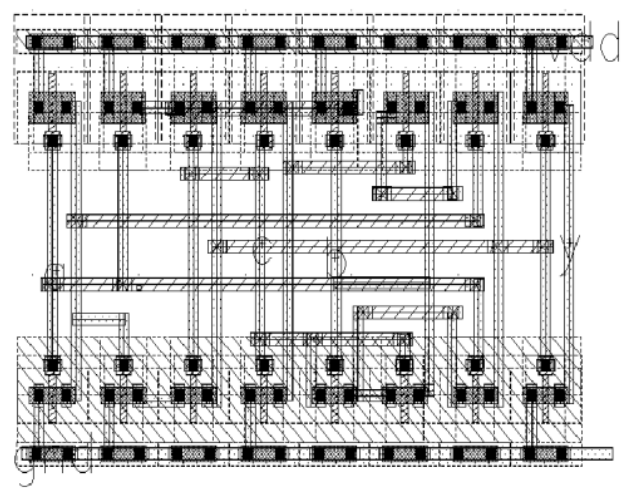

(b) The layout of the proposed $\mathrm{AND} / \mathrm{XOR}$ gate

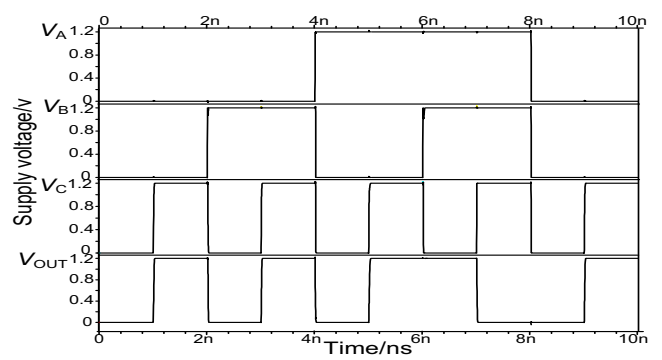

Figure 4. Transient waveform of proposed circuit

To compare the performance difference among the proposed gate and the published ones in Fig. 1, all of circuits are post-simulated under the same conditions. The average power consumption is measured during 100 operating cycles. The transient performances are characterized by $50 \%$ delay, which is defined as the time when the output voltage reaches the $50 \%$ of its steady state value. The PDP is used to evaluate the performance of the circuit. The improvements of power consumption, Delay and PDP are expressed by Power\%, Delay\% and $\mathrm{PDP} \%$, respectively. The comparison for circuits in Fig. 1 and Fig. 2 is shown in Table 1.

Figure 3. Test bench for three-input AND/XOR gates

Table 1. The electrical performance comparison for different gate circuits

\begin{tabular}{|c|c|c|c|c|c|c|}
\hline Circuits & Power $/ \mu \mathrm{w}$ & Delay/ns & PDP/ $\mu \mathrm{w} \cdot \mathrm{ns}$ & Power $\%$ & Delay $\%$ & PDP $\%$ \\
\hline CMOS & 2.46 & 0.22 & 0.54 & $19.51 \%$ & $4.54 \%$ & $22.22 \%$ \\
\hline TG & 2.72 & 0.23 & 0.63 & $27.21 \%$ & $8.70 \%$ & $33.33 \%$ \\
\hline HU & 2.50 & 0.26 & 0.65 & $20.08 \%$ & $19.23 \%$ & $35.39 \%$ \\
\hline LH & 1.99 & 0.25 & 0.50 & $0.50 \%$ & $16.00 \%$ & $16.00 \%$ \\
\hline Proposed & 1.98 & 0.21 & 0.42 & - & - & - \\
\hline
\end{tabular}

As shown in Table 1, the proposed circuit has the lowest PDP and the improvement ratio of PDP can be up to $35.39 \%$. The reason behind this is that the circuits like TG, HU and CMOS have cascaded structure, which increase the capacitance of internal nodes and power consumption and capacitance. Moreover, due to longer transmission path of cascaded structures, these circuits also have longer time delays. As the output of structure based on complementary CMOS is directly connected to power supply or ground, it has a good driving capability without the additional tailing inverter. For circuit LH, it has a larger delay because it needs pass the signal from the XOR to the transmission gate for some input patterns. In addition, the proposed circuit is the better and more competitive one.

In order to evaluate the capability of these circuits at different frequencies, the circuits are post-simulated at frequencies in the range from $100 \mathrm{MHZ}$ to $1400 \mathrm{MHZ}$ at $1.20 \mathrm{~V}$ supply voltage. The curves of power and PDP versus operation frequency in the range from $100 \mathrm{MHz}$ to 
1400MHZ are shown in Fig. 5(a) and (b). It can be seen that the proposed circuit can work reliably and has advantage in power and PDP.

The simulations are also carried out to check scaling capabilities of power supply voltages. Fig. 6 shows the

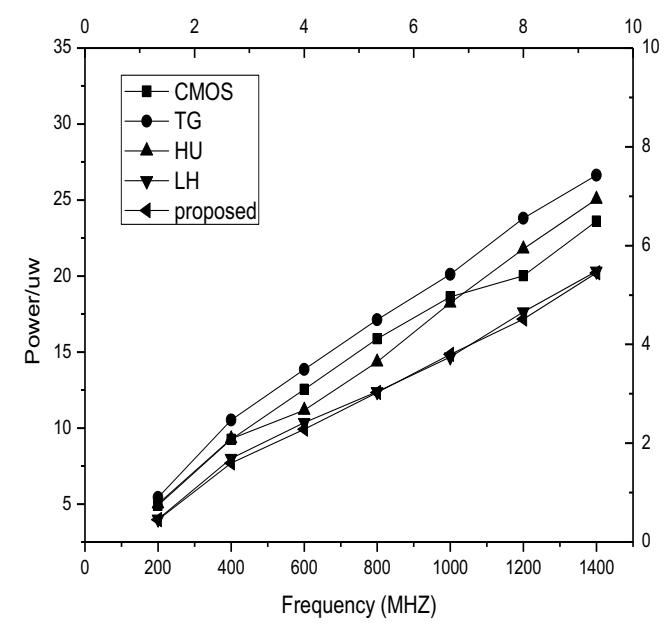

(a) Power consumption under TT process corner Figure 5. Performance under TT process corner

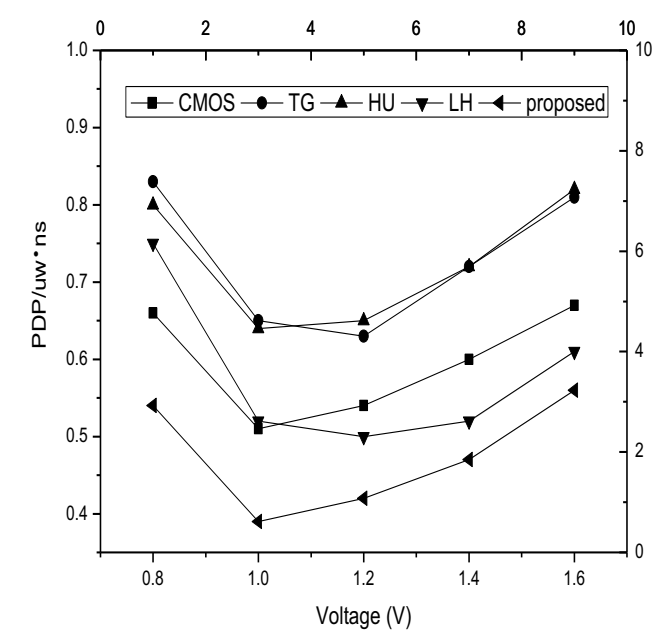

Figure 6. PDP versus supply voltage variatio

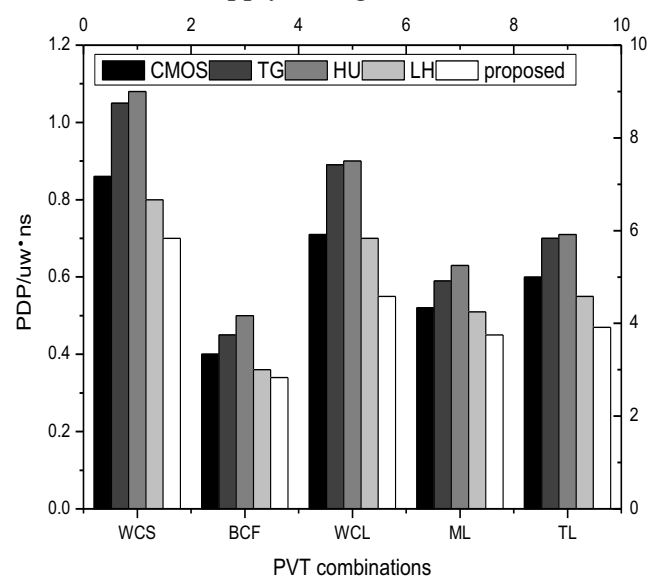

Figure 7. PDP under different PVT combinations

To further evaluate the effects of processes corners, voltage and temperature variations, post-simulations under different process corners are also carried out. Five standard STA conditions are chosen: (1) Worst Case Slow curve of PDP versus supply voltage varying from $1.6 \mathrm{~V}$ down to $0.8 \mathrm{~V}$ under $100 \mathrm{MHz}$. It can be seen that the proposed circuit has the best voltage scaling capability in terms of PDP.

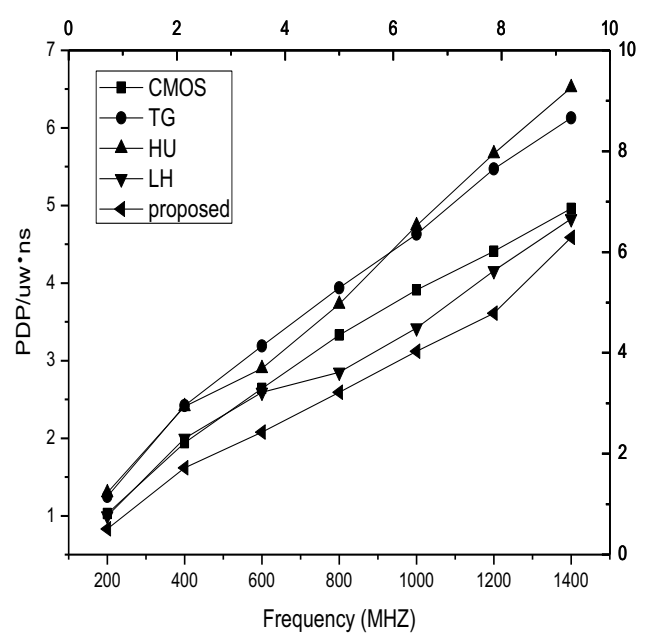

(b) PDP under TT process corner

(WCS): SS process corner, $125^{\circ} \mathrm{C}$ and $1.08 \mathrm{~V}$; (2) Best Case Fast (BCF): FF process corner, $-40^{\circ} \mathrm{C}$ and $1.32 \mathrm{~V}$; (3) Worst Case Cold (WCL): SS process corner, $-40^{\circ} \mathrm{C}$ and $1.08 \mathrm{~V}$; (4) Maximal Leakage (ML): FF process corner, $125^{\circ} \mathrm{C}$ and $1.32 \mathrm{~V}$; (5) TL (Typical Leakage): TT process corner, $125^{\circ} \mathrm{C}$ and $1.20 \mathrm{~V}$. Simulation results are shown in Fig. 7. It is observed that the proposed gate circuit has the lowest PDP in various cases, demonstrating the advantage and feasibility in hybrid-CMOS logic.

\section{Conclusion}

In this paper, a transistor level 3-input AND/XOR gate is proposed. Multiple transimission paths are employed to elimate the threshold voltage loss and improve its performance. Moreover, in order to decrease its delay, the hybrid-CMOS techniques are used to short the signal transimission path. The 16T 3-input AND/XOR gate is post-simulated under $55 \mathrm{~nm}$ CMOS process, and the results are compared with four published circuits in terms of power consumption, delay and PDP. Comparison shows the proposed AND/XOR gate has the lowest PDP compared with those published AND/XOR gates and the improvement ratio of PDP can be up to $35.39 \%$. Moreover, the post-simulations under five different PVT combinations are also carried out and show that the proposed circuit has the lowest PDP.

\section{References}

1. Y. S. Xia, K.Y. Mao, X.E. Ye, Journal of ComputerAided Design \& Computer Graphics, 19, 15221527(2007)

2. L.Y. Wang, Y.S. Xia, X.X. Cheng, Journal of Computer-Aided Design \& Computer Graphics, 24,961-967 (2012) 
3. M.H. Moaiyeri, R. F. Mirzaee, K. Navi, et al, International Journal of Electronics, 97, 647662(2010)

4. S. Purohit, M. Margala, IEEE Transactions on Very Large Scale Integration (VLSI) Systems, 20 ,13271331(2012)

5. S. Wairya, G. Singh, Vishant, et al, Proceedings of IEEE International Conference on Engineering ,17(2011)

6. J.P. Hu, Z.L. Li, Y.S. Xia. Energy Education Science and Technology Part A: Energy Science and Research, 30, 85-92(2012)
7. N. Zhuang, H.M. Wu. IEEE Journal of Solid-state Circuits, 27,840-844(1992)

8. S.S. Mishra, A.K. Agrawal, R.K. Nagaria. International Journal on Emerging Technologies, 1,1-10(2010)

9. S. Nishizawa, T. Ishihara, H. Onodera. Proceedings of IEEE 14th International Symposium on Quality Electronic Design,703-708 (2013)

10. H. Liang, Y.S. Xia, L.B. Qian, et al, Journal of computer-Aided Design \& Computer Graphics, 27, 940-945 (2015) 